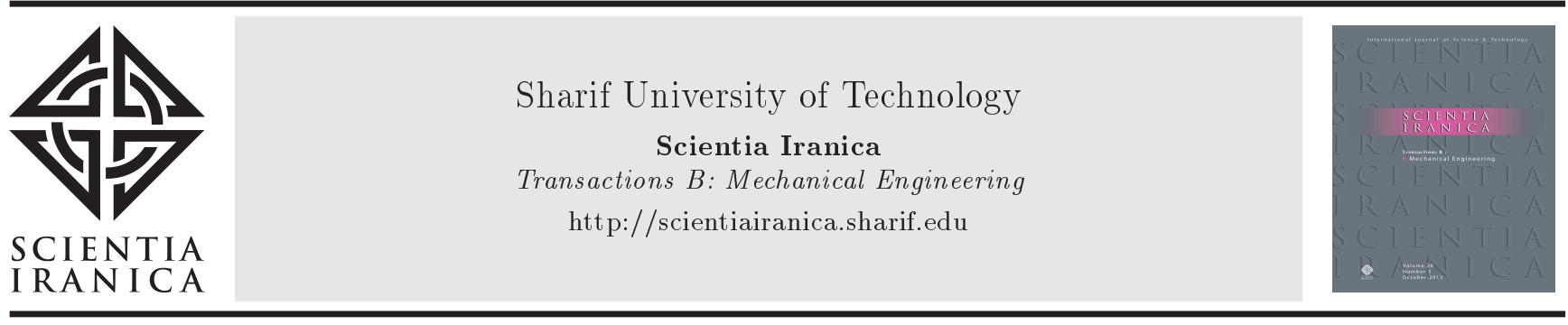

\title{
Mode III fracture analysis of a non-homogeneous layer bonded to an elastic half-plane weakened by multiple interface cracks
}

\author{
R. Sourki ${ }^{a}$, M. Ayatollahi ${ }^{a, *}$, and M.M. Monfared ${ }^{b}$ \\ a. Faculty of Engineering, University of Zanjan, Zanjan, P.O. Box 45195-313, Iran. \\ b. Department of Mechanical Engineering, Hashtgerd Branch, Islamic Azad University, P.O. Box 33615-178, Alborz, Iran.
}

Received 11 November 2016; received in revised form 8 June 2017; accepted 11 September 2017

\author{
KEYWORDS \\ Interface cracks; \\ Distributed \\ dislocation technique; \\ Functionally graded \\ layer; \\ Volterra type screw \\ dislocation; \\ Stress intensity \\ factors.
}

\begin{abstract}
In this paper, the mode III crack problem of a non-homogenous layer bonded to an elastic half-plane was considered. It was assumed that the half-plane is homogeneous and the layer is non-homogeneous where the elastic properties are continuous throughout the layer. The stress field in a non-homogeneous layer and in an elastic half-plane with Volterratype screw dislocation was obtained. Fourier transforms were applied to the governing equations to derive a system of singular integral equations with simple Cauchy kernel. Then, the integral equations were solved numerically by being converted into a system of linear algebraic equations and using a collocation technique. The given results demonstrate the effects of the non-homogeneity parameters, interaction between the multiple cracks, and distance of the cracks in the stress intensity factors for gaining better understanding of the mechanical behavior of the non-homogenous coating.
\end{abstract}

(C) 2018 Sharif University of Technology. All rights reserved.

\section{Introduction}

Functionally Graded Materials (FGMs) with material properties varying continuously possess apparent advantages in maintaining the integrity of the structure when subjected to applied thermo-mechanical loads. They are also widely used in engineering fields as a thermal barrier to prevent chemical corrosion [1]. Functionally Graded Materials (FGMs) have been widely applied under extreme loading environment, and the fracture mechanics of FGMs have attracted much attention [2,3]. Practical application shows that

\footnotetext{
*. Corresponding author. Tel.: +982433052488;

Fax: +98241228-3204

E-mail addresses: rsourki@gmail.com (R. Sourki);

mo.ayatollahy@gmail.com (M. Ayatollahi);

mo_m_monfared@yahoo.com (M.M. Monfared).
}

doi: $10.24200 /$ sci. 2017.4493 interface cracks constitute a major reason for failures of structural connection of different materials. Thus, the study of the stress field of the interface is playing a significant role in the design of safe layered structures. In addition, due to the nature of the techniques used in processing, these materials are imperfect. Moreover, by far, the most common forms of such imperfections, which may have the potential of growing into a macroscopic crack and causing the failure of the structure eventually, are the surface flaws located in the intersection of the interfaces [4].

In fracture analysis of mediums with interfacial cracks, Erdogan is an expert as one of the pioneering researchers; therefore, a concise review regarding his contributions in this category is presented herein at first. The analytical expression of Stress Intensity Factor (SIFs) in two bonded elastic layers containing cracks perpendicular to the interface was obtained by Ming-Che and Erdogan [4]. The problem was 
formulated in terms of integral equations and the singular behavior of the solution near to and at the ends of intersection of the cracks. The solution to an interface crack between two bonded homogeneous and non-homogeneous half planes was presented by Delale and Erdogan [5]. The results led to the conclusion that the singular behavior of stresses in the nonhomogeneous medium is identical to that in a homogeneous material. In another paper, Erdogan and $\mathrm{Wu}$ [6] investigated the influence of the structure and thickness of the interfacial regions on the strain energy release rate in bonded isotropic or orthotropic materials containing collinear interface cracks. They formulated the problem in terms of a system of singular integral equations of the second kind. The results show that the effects of properties and the relative thickness of the interfacial region on the stress intensity factors and strain energy release rate can be highly significant. Erdogan et al. [7] solved the mode III crack problem for two bonded homogeneous half planes. The interfacial zone was modelled by a non-homogeneous strip in such a way that the shear modulus is a continuous function throughout the composite medium and has discontinuous derivatives along the boundaries of the interfacial zone. It was shown that the stresses had the standard square root singularity.

Besides, in recent years, there have been several investigations regarding mode III interface crack problems as well. For example, Lü et al. [8] investigated the asymmetrical dynamic propagation problems of mode III interface crack under the condition of point loads and unit-step loads by the application of the theory of complex functions. The problem of a functionally graded coating bonded to an elastic substrate with interface crack subject to anti-plane loading was analyzed by Ding and $\mathrm{Li}$ [9]. In this investigation, they studied the influence of the interaction between the periodic arrays of interface cracks on stress intensity factors. Chen and Chue [10] dealt with the anti-plane problems of two bonded FGM strips weakened by an internal crack normal to the interface. The material properties are assumed to vary along the direction of the crack lines. The derived system of singular integral equations was solved numerically by Gauss-Chebyshev integration formula. Chue and Yeh [11] considered the anti-plane crack problem of two bonded FGM strips. Each strip concludes an arbitrarily oriented crack, and the material properties are assumed to be in exponential forms. Mode III crack cutting the interface perpendicularly between two dissimilar semiinfinite magneto-electro-elastic solids was investigated by Wan et al. [12]. The problem was formulated and solved based on the complex variable method, and the analytical solution was then found for the entire plane. Chao and Lu [13] studied the problem of a layered structure demonstrating an arbitrary oriented crack crossing the interface in anti-plane elasticity. The distributed dislocation was used to model a crack, and SIFs for various inclinations with different material properties were found. Pan et al. [14] investigated the fracture problem of multiple parallel symmetric and permeable mode III cracks in a FG piezoelectric material plane under anti-plane loadings. They have found that the SIFs of cracks depend on the crack length, FG parameter, and the distance between the parallel cracks. Ding and $\mathrm{Li}$ [15] investigated an anti-plane crack problem of two collinear cracks perpendicular to the interface of a FG orthotropic layer bonded to an orthotropic homogeneous substrate. Choi [16] provided the solution to the anti-plane problem of dissimilar nonhomogeneous layers weakened by an embedded or edge interfacial crack. Ordyan and Petrova [17] presented a solution to the interaction of an interface crack with internal cracks in dissimilar materials under anti-plane shear loading. The fracture behavior of a piezoelectro-magneto-elastic medium under anti-plane loading was analyzed by Rogowski [18]. It was found that the stress, electric and magnetic fields exhibit square root singularity at the crack tips. Lapusta et al. [19] analyzed an interface crack in a biomaterial piezoelectric plane under anti-plane mechanical and inplane electric loadings.

Lï et al. [20] investigated the asymmetrical dynamic propagation problem on the edges of mode III interface crack subjected to superimposed loads by application of the theory of complex variable functions. $\mathrm{Hu}$ et al. [21] investigated a moving Dugdale interfacial crack model between dissimilar magneto-electro-elastic materials under anti-plane shear and in-plane electric and magnetic loadings. Monfared and Ayatollahi [22] investigated the dynamic stress intensity factors of cracked orthotropic half-plane and functionally graded material coating of a coating-substrate material due to the action of anti-plane traction on the crack surfaces. The dislocation solution was utilized to derive integral equations for multiple interacting cracks. The transient response analysis of a mode III interface crack between a piezoelectric layer and a FG orthotropic material was carried out by Shin and Kim [23].

Many researchers have also studied the mixed mode interfacial crack problems in recent years. For instance, Yang et al. [24] studied the fracture problems near the interface crack tip of double dissimilar orthotropic composite materials. Through the instrumentality of complex function method, the singularity exponents were derived and determined. Cheng et al. [25] studied the plane elasticity problem of two bonded dissimilar functionally graded strips containing an interface crack with material properties varying arbitrarily. The governing equation in terms of Airy stress function was formulated, and exact solutions were obtained for several special variations of material 
properties in Fourier transformation domain. The fatigue crack growth of interfacial cracks in bi-layered materials using the extended finite-element method was analysed by Bhattacharya et al. [26]. Ding et al. [27] studied the behavior of an interface crack for a homogeneous orthotropic strip sandwiched between two various FG orthotropic materials subjected to thermomechanical loading. Crack tip opening displacement and plastic zone size were investigated for a curved interface crack between a circular inclusion and an infinite matrix by Fan et al. [28]. Zhao et al. [29] studied the stress intensity factor in orthotropic bimaterial interface cracks adopting the complex function approach .

Moreover, there have been several investigations using numerical techniques to solve such problems. For instance, Bui and Zhang [30] presented a transient dynamic analysis of stationary cracks in two-dimensional solids subjected to coupled electromechanical impact loads using the extended Finite-Element Method (XFEM). Sharma et al. [31] analyzed a sub-interface crack problem in piezoelectric biomaterials by the X-FEM. In this investigation, the effects of different poling directions and electromechanical impact loads were analyzed. The stress intensity factors were evaluated by utilizing the asymptotic crack-tip fields. Liu et al. [32] studied the transient dynamic fracture behaviors of stationary cracked FG piezoelectric materials under impact loading using the X-FEM. The transient thermal shock fracture analysis of functionally graded piezoelectric materials using the X-FEM was conducted by Liu et al. [33]. It was found that the effect of the thermal shock loading on the dynamic intensity factors was significant. Yu et al. [34] solved the problem of interfacial cracks between dissimilar piezoelectric solids under coupled electromechanical impact loadings by the X-FEM. Bui [35] presented an extension of the extended isogeometric analysis to simulate two-dimensional fracture mechanics problems in piezoelectric materials under dynamic and static coupled electromechanical loading conditions. Bui et al. [36] developed a dynamic extended Iso Geometric Analysis (XIGA) of transient fracture of cracked magneto-electroelastic solids under coupled electromagneto-mechanical loading. Doan et al. [37] presented a numerical simulation of dynamic crack propagation in functionally graded glass-filled epoxy beams by utilizing a regularized variation formulation. The Griffith theory-based hybrid phase filed approach was used to simulate the dynamic crack growth accurately. Wang and Waisman [38] proposed a set of enrichment functions within the framework of the X-FEM for the analysis of interface cracks in biomaterials. Itou [39] determined the stress intensity factors for four collinear interface cracks which are situated at the interface between a nonhomogeneous elastic bonding layer and one of two dissimilar elastic half-planes. The behavior of an interface crack in two bonded dissimilar materials subjected to in-plane loading was studied by Monfared et al. [40]. The dislocation density on the faces of the cracks was obtained numerically and, then, was used to calculate the mixed mode stress intensity factors of multiple interface cracks.

In this article, we present an analytical method to study multiple interfacial cracks with arbitrary arrangement under anti-plane shear loading. This technique is efficient and applicable to modelling the evolution of a developing crack in two or three dimensions. Basically, the same strategies will be employed to formulate the solution to three-dimensional crack problems by dislocation loop instead of a straight line dislocation.

This paper presents the solution to the problem of a graded coating bonded to an elastic isotropic halfplane weakened by multiple interface cracks. The stress fields in a medium containing Volterra-type screw dislocation are first presented (Section 2). The analytical study is based on the use of Fourier transform. In the next section, the stress analysis of a medium under point loading is carried out (Section 3 ). With respect to the results of Sections 2 and 3, the integral equations for multiple interface cracks are derived in terms of the dislocation density function given in Section 4. Several examples are solved to demonstrate the applicability of the problem presented in Section 5. Numerical examples are given to show the effects of the thickness of the FGM strips, material properties, and length of the crack upon the fracture behavior. Finally, Section 6 will conclude the paper.

\section{Formulation of the problem}

The anti-plane problem under consideration consists of a graded coating bonded to isotropic semi-infinite substrate with an interface crack (see Figure 1). The thickness of strip is $h$ in $y$-direction, where shear modulus, $\mu$, varies continuously in the thickness direction. There is a screw dislocation at $y=0$ between the graded coating and elastic substrate. The constitutive relations for FGM and isotropic material may be expressed as follows:

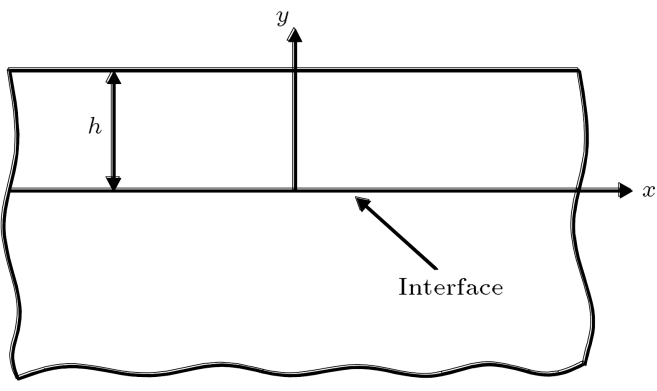

Figure 1. Geometry of the problem. 


$$
\begin{aligned}
\sigma_{z x 1}=\mu(y) \frac{\partial w_{1}}{\partial x}, & \sigma_{z y 1}=\mu(y) \frac{\partial w_{1}}{\partial y}, \\
\sigma_{z x 2}=\mu_{0} \frac{\partial w_{2}}{\partial x}, & \sigma_{z y_{2}}=\mu_{0} \frac{\partial w_{2}}{\partial y} .
\end{aligned}
$$

To facilitate the solution of the problem, nonhomogeneity of the material may be approximated by:

$$
\mu(y)=\mu_{0} e^{2 \beta y}
$$

where $\beta$ is a non-homogeneity parameter. In the above equations, $\mu_{0}$ is the shear module of elasticity. The strip is traction free on the boundary. Thus, boundary condition is as follows:

$$
\sigma_{z y_{1}}(x, h)=0 \text {. }
$$

A Volterra-type screw dislocation with Burgers vector; $b_{z}$, is placed at the origin of coordinates. The condition illustrating the dislocation is:

$$
w_{1}\left(x, 0^{+}\right)-w_{2}\left(x, 0^{-}\right)=b_{z} H(x)
$$

where $H($.$) is the Heaviside step function. The condi-$ tion of self-equilibrium of stress between the strip and half-plane implies that:

$$
\sigma_{z y_{1}}\left(x, 0^{+}\right)=\sigma_{z y_{2}}\left(x, 0^{-}\right) .
$$

The equilibrium equations in terms of displacement will be written as follows:

$$
\begin{array}{ll}
\frac{\partial^{2} w}{\partial x^{2}}+\frac{\partial^{2} w}{\partial y^{2}}+2 \beta \frac{\partial w}{\partial y}=0, & 0 \leq y \leq h, \\
\frac{\partial^{2} w}{\partial x^{2}}+\frac{\partial^{2} w}{\partial y^{2}}=0, & y \leq 0 .
\end{array}
$$

The solution to Eqs. (6) can be solved by the Fourier transformation, defined as follows:

$$
F(s)=\int_{-\infty}^{\infty} f(x) e^{i s x} d x
$$

and the inversion of the Fourier transform is:

$$
f(x)=\frac{1}{2 \pi} \int_{-\infty}^{\infty} F(s) e^{-i s x} d s
$$

By applying the Fourier transform Eq. (7), the solution to Eqs. (6) may be expressed as follows:

$$
\begin{aligned}
& \frac{d^{2} W(s, y)}{d y^{2}}+2 \beta \frac{d W(s, y)}{d y} \\
&-s^{2} W(s, y)=0, \quad 0 \leq y \leq h, \\
& \frac{d^{2} W(s, y)}{d y^{2}}-s^{2} W(s, y)=0, \quad y \leq 0,
\end{aligned}
$$

where $s$ is the transform variable, and the solution will lead to the following equations for each region:

$$
\begin{aligned}
W(s, y)= & A_{1}(s) e^{(-\beta-\alpha) y}+A_{2}(s) e^{(-\beta+\alpha) y} \\
& 0 \leq y \leq h, \\
W(s, y)= & B(s) e^{|s| y}, \quad y \leq 0,
\end{aligned}
$$

where $\alpha=\sqrt{\beta^{2}+s^{2}}$ and unknown functions, $A_{1}(s)$, $A_{2}(s)$, and $B(s)$ are determined from the boundary conditions. The Fourier transforms of boundary conditions (3) to (5) result in:

$$
\begin{aligned}
& \frac{d W_{1}(s, h)}{d y}=0 \\
& W_{1}\left(s, 0^{+}\right)-W_{2}\left(s, 0^{-}\right)=b_{z}\left(\pi \delta(s)+\frac{i}{s}\right), \\
& \frac{d W_{1}\left(s, 0^{+}\right)}{d y}=\frac{d W_{2}\left(s, 0^{-}\right)}{d y}
\end{aligned}
$$

where $\delta($.$) is the Dirac delta function. The solution to$ Eq. (10) subject to the above boundary conditions is straightforward. Hence, unknowns $A_{1}(s), A_{2}(s)$, and $B_{1}(s)$ may be expressed as shown in Box I.

By substituting Eq. (12) into Eq. (10) and applying the Fourier transform inversion formula (8), the

$$
\begin{aligned}
& A_{1}(s)=-\frac{b_{z}|s|(\pi \delta(s)+i / s)(\beta-\alpha) e^{\alpha h}}{|s|\left[(\beta+\alpha) e^{-\alpha h}-(\beta-\alpha) e^{\alpha h}\right]+\left(\beta^{2}-\alpha^{2}\right)\left(e^{-\alpha h}-e^{\alpha h}\right)}, \\
& A_{2}(s)=\frac{b_{z}|s|(\pi \delta(s)+i / s)(\beta+\alpha) e^{-\alpha h}}{|s|\left[(\beta+\alpha) e^{-\alpha h}-(\beta-\alpha) e^{\alpha h}\right]+\left(\beta^{2}-\alpha^{2}\right)\left(e^{-\alpha h}-e^{\alpha h}\right)} \\
& B_{1}(s)=\frac{b_{z}(\pi \delta(s)+i / s)\left(\beta^{2}-\alpha^{2}\right)\left(e^{\alpha h}-e^{-\alpha h}\right)}{|s|\left[(\beta+\alpha) e^{-\alpha h}-(\beta-\alpha) e^{\alpha h}\right]+\left(\beta^{2}-\alpha^{2}\right)\left(e^{-\alpha h}-e^{\alpha h}\right)}
\end{aligned}
$$




$$
\begin{aligned}
& w_{1}(x, y)=-\frac{b_{z}}{2 \pi} \int_{-\infty}^{\infty} \frac{|s|(\pi \delta(s)+i / s) e^{-\beta y}\left[(\beta-\alpha) e^{\alpha(h-y)}-(\beta+\alpha) e^{-\alpha(h-y)}\right]}{|s|\left[(\beta+\alpha) e^{-\alpha h}-(\beta-\alpha) e^{\alpha h}\right]+\left(\beta^{2}-\alpha^{2}\right)\left(e^{-\alpha h}-e^{\alpha h}\right)} e^{-i s x} d s, \quad 0 \leq y \leq h \\
& w_{2}(x, y)=\frac{b_{z}}{2 \pi} \int_{-\infty}^{\infty} \frac{(\pi \delta(s)+i / s)\left(\beta^{2}-\alpha^{2}\right)\left(e^{\alpha h}-e^{-\alpha h}\right) e^{|s| y}}{|s|\left[(\beta+\alpha) e^{-\alpha h}-(\beta-\alpha) e^{\alpha h}\right]+\left(\beta^{2}-\alpha^{2}\right)\left(e^{-\alpha h}-e^{\alpha h}\right)} e^{-i s x} d s, \quad y \leq 0 .
\end{aligned}
$$

\section{Box II}

displacement field in the medium can be obtained by Eq. (13) as shown in Box II.

By splitting the integrals in Eq. (13) into odd and even parts with respect to parameter $s$ in view of Eq. (1), the stress fields are written as follows:

$$
\begin{aligned}
& \sigma_{z y 1}(x, y)=-\frac{b_{z} \mu_{0} e^{\beta y}}{\pi} \int_{0}^{\infty} \\
& \frac{s\left(e^{\alpha(h-y)}-e^{-\alpha(h-y)}\right)}{(\beta+\alpha-s) e^{-\alpha h}-(\beta-\alpha-s) e^{\alpha h}} \sin (s x) d s, \\
& \sigma_{z x 1}(x, y)=-\frac{b_{z} \mu_{0} e^{\beta y}}{\pi} \int_{0}^{\infty} \\
& \frac{(\beta-\alpha) e^{\alpha(h-y)}-(\beta+\alpha) e^{-\alpha(h-y)}}{(\beta+\alpha-s) e^{-\alpha h}-(\beta-\alpha-s) e^{\alpha h}} \cos (s x) d s,
\end{aligned}
$$$$
0 \leq y \leq h
$$$$
\sigma_{z y 2}(x, y)=-\frac{b_{z} \mu_{0}}{\pi} \int_{0}^{\infty}
$$$$
\frac{s e^{s y}\left(e^{\alpha h}-e^{-\alpha h}\right)}{(\beta+\alpha-s) e^{-\alpha h}-(\beta-\alpha-s) e^{\alpha h}} \sin (s x) d s,
$$$$
\sigma_{z x 2}(x, y)=-\frac{b_{z} \mu_{0}}{\pi} \int_{0}^{\infty}
$$$$
\frac{s e^{s y}\left(e^{\alpha h}-e^{-\alpha h}\right)}{(\beta+\alpha-s) e^{-\alpha h}-(\beta-\alpha-s) e^{\alpha h}} \cos (s x) d s,
$$$$
y \leq 0 \text {. }
$$

In order to specify the singular nature of the stress components, the asymptotic behavior of integrands in Eq. (14) should be examined. Since the integrands are continuous functions of $s$ and also finite at $s=0$, the singularity must occur as $s$ tends to infinity. The final results may be presented as follows:

$$
\begin{aligned}
& \sigma_{z y 1}(x, y)= \\
& -\frac{\mu_{0} b_{z} e^{2 \beta y}}{2 \pi}\left(\frac{x}{x^{2}+y^{2}}+\frac{x}{x^{2}+(2 h-y)^{2}}\right)-\frac{\mu_{0} b_{z} e^{\beta y}}{\pi} \\
& \times \int_{0}^{\infty}\left[\frac{s\left(e^{\alpha h} e^{-(\beta+\alpha) y}-e^{-\alpha h} e^{-(\beta-\alpha) y}\right)}{(\beta+\alpha-s) e^{-\alpha h}-(\beta-\alpha-s) e^{\alpha h}}\right. \\
& \left.-\frac{e^{-s y}-e^{-s(2 h-y)}}{2}\right] \sin (s x) d s, \\
& 0 \leq y \leq h \\
& \sigma_{z x 1}(x, y)=\frac{\mu_{0} b_{z} e^{2 \beta y}}{2 \pi}\left(\frac{y}{x^{2}+y^{2}}+\frac{2 h-y}{x^{2}+(2 h-y)^{2}}\right) \\
& -\frac{\mu_{0} b_{z} e^{\beta y}}{\pi} \\
& \times \int_{0}^{\infty}\left[\frac{(\beta-\alpha) e^{\alpha h} e^{-(\beta+\alpha) y}-(\beta+\alpha) e^{-\alpha h} e^{-(\beta-\alpha) y}}{(\beta+\alpha-s) e^{-\alpha h}-(\beta-\alpha-s) e^{\alpha h}}\right. \\
& \left.+\frac{e^{-s y}-e^{-s(2 h-y)}}{2}\right] \cos (s x) d s, \\
& 0 \leq y \leq h \\
& \sigma_{z y 2}(x, y)=-\frac{\mu_{0} b_{z}}{2 \pi}\left(\frac{x}{x^{2}+y^{2}}\right)-\frac{\mu_{0} b_{z}}{\pi} \int_{0}^{\infty} \\
& {\left[\frac{s\left(e^{\alpha h}-e^{-\alpha h}\right)}{(\beta+\alpha-s) e^{-\alpha h}-(\beta-\alpha-s) e^{\alpha h}}-\frac{1}{2}\right] e^{s y} \sin (s x) d s,} \\
& y \leq 0 \\
& \sigma_{z x 2}(x, y)=\frac{\mu_{0} b_{z}}{2 \pi}\left(\frac{x}{x^{2}+y^{2}}\right)-\frac{\mu_{0} b_{z}}{\pi} \int_{0}^{\infty} \\
& {\left[\frac{s\left(e^{\alpha h}-e^{-\alpha h}\right)}{(\beta+\alpha-s) e^{-\alpha h}-(\beta-\alpha-s) e^{\alpha h}}-\frac{1}{2}\right] e^{s y} \cos (s x) d s,}
\end{aligned}
$$$$
y \leq 0 \text {. }
$$ 
It is noteworthy to mention that stress fields exhibit Cauchy singularity at the dislocation position. Moreover, the integrands in Eq. (15) decay quite rapidly as $s \rightarrow \infty$, which makes the integrals susceptible to numerical evaluation.

\section{Point load solution}

It is assumed that the medium is under an antiplane point load with magnitude $\tau_{0}$ on the boundary, represented by the following:

$$
\sigma_{z y_{1}}(x, h)=\tau_{0} \delta(x) .
$$

The continuity conditions can be expressed as follows:

$$
\begin{aligned}
& w_{1}\left(x, 0^{+}\right)=w_{2}\left(x, 0^{-}\right), \\
& \sigma_{z y_{1}}\left(x, 0^{+}\right)=\sigma_{z y_{2}}\left(x, 0^{-}\right) .
\end{aligned}
$$

The expressions for the displacements and stresses may be obtained as shown in Box III.

The singular parts of the kernels in Eq. (18) may be evaluated by separating the leading terms in the asymptotic analysis for $s \rightarrow \infty$. Thus, after performing the appropriate asymptotic analysis using a symbolic manipulator and separating the singular parts of the kernels, we obtain:

$$
\begin{aligned}
\sigma_{z y_{1}}(x, y)= & \frac{\tau_{0} e^{\beta(h-y)}}{\pi}\left\{\frac{(h-y)}{x^{2}+(h-y)^{2}}\right. \\
& +\int_{0}^{\infty}\left[\frac{(\beta+\alpha-s) e^{-\alpha y}-(\beta-\alpha-s) e^{\alpha y}}{(\beta+\alpha-s) e^{-\alpha h}-(\beta-\alpha-s) e^{\alpha h}}\right. \\
& \left.\left.-e^{-s(h-y)}\right] \cos (s x) d s\right\}, \quad 0 \leq y \leq h,
\end{aligned}
$$

$$
\begin{aligned}
\sigma_{z x 1}(x, y)= & -\frac{\tau_{0} e^{\beta(h-y)}}{\pi}\left\{\frac{x}{x^{2}+(h-y)^{2}}\right. \\
& +\int_{0}^{\infty}\left[\frac{(\beta+\alpha-s) e^{-\alpha y}-(\beta-\alpha-s) e^{\alpha y}}{(\beta+\alpha-s) e^{-\alpha h}-(\beta-\alpha-s) e^{\alpha h}}\right. \\
& \left.\left.-e^{-s(h-y)}\right] \sin (s x) d s\right\}, \quad 0 \leq y \leq h,
\end{aligned}
$$

$$
\begin{aligned}
& w_{1}(x, y)=\frac{\tau_{0}}{2 \pi \mu_{0}} \int_{-\infty}^{\infty} \frac{e^{(\beta+\alpha)(h-y)-2 \beta y}\left[(\beta+\alpha+|s|) e^{2 \alpha y}-(\beta-\alpha+|s|)\right]}{s^{2}\left(e^{2 \alpha h}-1\right)+|s|\left[(\beta+\alpha)-(\beta-\alpha) e^{2 \alpha h}\right]} e^{-i s x} d s \\
& 0 \leq y \leq h, \\
& w_{2}(x, y)=\frac{\tau_{0}}{\pi \mu_{0}} \int_{-\infty}^{\infty} \frac{\alpha e^{(|s|-2 \beta) y+(\beta+\alpha) h}}{\left(1-e^{2 \alpha h}\right)\left(\beta^{2}-\alpha^{2}\right)+|s|\left[\alpha\left(1+e^{2 \alpha h}\right)+\beta\left(1-e^{2 \alpha h}\right)\right]} e^{-i s x} d s, \\
& y \leq 0, \\
& \sigma_{z y 1}(x, y)=\frac{\tau_{0} e^{\beta(h-y)}}{\pi} \int_{0}^{\infty} \frac{(\beta+\alpha-s) e^{-\alpha y}-(\beta-\alpha-s) e^{\alpha y}}{(\beta+\alpha-s) e^{-\alpha h}-(\beta-\alpha-s) e^{\alpha h}} \cos (s x) d s, \\
& 0 \leq y \leq h, \\
& \sigma_{z x 1}(x, y)=-\frac{\tau_{0} e^{\beta(h-y)}}{\pi} \int_{0}^{\infty} \frac{(\beta+\alpha-s) e^{-\alpha y}-(\beta-\alpha-s) e^{\alpha y}}{(\beta+\alpha-s) e^{-\alpha h}-(\beta-\alpha-s) e^{\alpha h}} \sin (s x) d s \\
& 0 \leq y \leq h, \\
& \sigma_{z y_{2}}(x, y)=\frac{2 \tau_{0}}{\pi} \int_{0}^{+\infty} \frac{\alpha(s-2 \beta) e^{\beta(h-2 y)+s y}}{s\left[(\beta+\alpha-s) e^{-\alpha h}-(\beta-\alpha-s) e^{\alpha h}\right]} \cos (s x) d s, \\
& y \leq 0, \\
& \sigma_{z x_{2}}(x, y)=-\frac{2 \tau_{0}}{\pi} \int_{0}^{\infty} \frac{\alpha e^{\beta(h-2 y)+s y}}{(\beta+\alpha-s) e^{-\alpha h}-(\beta-\alpha-s) e^{\alpha h}} \sin (s x) d s \\
& y \leq 0 .
\end{aligned}
$$




$$
\begin{aligned}
\sigma_{z y 2}(x, y)= & \frac{\tau_{0} e^{\beta(h-2 y)}}{\pi}\left\{\frac{(h-y)}{x^{2}+(h-y)^{2}}\right. \\
& +\int_{0}^{\infty}\left[\frac{2 \alpha(s-2 \beta)}{s\left[(\beta+\alpha-s) e^{-\alpha h}-(\beta-\alpha-s) e^{\alpha h}\right]}\right. \\
& \left.\left.-e^{-s h}\right] e^{s y} \cos (s x) d s\right\}, \quad y \leq 0, \\
\sigma_{z x 2}(x, y)= & -\frac{\tau_{0} e^{\beta(h-2 y)}}{\pi}\left\{\frac{x}{x^{2}+(h-y)^{2}}\right. \\
& +\int_{0}^{\infty}\left[\frac{2 \alpha}{(\beta+\alpha-s) e^{-\alpha h}-(\beta-\alpha-s) e^{\alpha h}}\right. \\
& \left.\left.-e^{-s h}\right] e^{s y} \sin (s x) d s\right\}, \quad y \leq 0, \quad(19)
\end{aligned}
$$

At the points of application of load, i.e., $r \rightarrow 0$, stress fields exhibit the familiar Cauchy type singularity, $1 / r$, where $r$ is the distance from the load.

\section{Medium weakened by crack}

The dislocation solutions accomplished in the preceding section may be employed to analyze several arbitrarily cracks located in the interface of two dissimilar mediums. A crack configuration with respect to Cartesian coordinate $x-y$ may be described in a parametric form as follows:

$$
\begin{aligned}
& x_{i}=x_{i 0}+a_{i} \eta, \\
& y_{i}=y_{i 0}, \quad i=1,2, \ldots, N, \quad-1 \leq \eta \leq 1,
\end{aligned}
$$

where $\left(x_{i 0}, y_{i 0}\right)$ is the coordinates of the $i$ th crack center. Suppose that the screw dislocation unknown density, $B_{z i}(t)$, is distributed on segment $a_{i} d t$ at the surface of the $i$ th crack, where $-1 \leq t \leq 1$. Covering the cracks surfaces by dislocations, the principal superposition may be invoked to obtain traction on the given crack surface. The anti-plane field traction on the face of the $i$ th crack due to the presence of distribution of the above-mentioned dislocation on all $N$ cracks is obtained. The system of a singular equation can be written in the following form utilized in a numerical procedure:

$$
\begin{gathered}
\sigma_{z y}\left(x_{i}(\eta), y_{i}(\eta)\right)=\sum_{j=1}^{N} \int_{-1}^{1} k_{i j}(\eta, t) a_{j} B_{z j}(t) d t, \\
-1 \leq \eta \leq 1, \quad i=1,2, \ldots, N .
\end{gathered}
$$

Kernel $K_{i j}$ in integral Eqs. (21) is coefficient of $b_{z}$ in the stress components. By virtue of the Buckner's principle [41], the left-hand side of Eqs. (21), after changing the sign, is the traction caused by the point load given in Section 3. The equation for the crack opening displacement across the $i$ th crack is as follows:

$$
w_{1 i}(\eta)-w_{2 i}(\eta)=\int_{-1}^{\eta} a_{i} B_{z i}(t) d t, \quad i \in\{1,2, \ldots, N\} .
$$

The displacement field is single-valued out of crack surfaces. Consequently, the dislocation density is subjected to the following closure requirement:

$$
\int_{-1}^{1} B_{z i}(t) d t=0, \quad i \in\{1,2, \ldots, N\}
$$

The stress fields in the neighborhood of crack tips behave like $1 / \sqrt{r}$, where $r$ is the distance from the crack tip. Therefore, the dislocation densities are taken as follows:

$$
\begin{aligned}
B_{z i}(t) & =\frac{g_{z i}(t)}{\sqrt{1-t^{2}}}, \\
-1 & \leq t \leq 1, \quad i \in\{1,2, \ldots, N\} .
\end{aligned}
$$

Parameters $g_{z i}(t)$ are obtained by solving the system of Eqs. (21) and (24) using numerical solutions of integral equations with Cauchy-type kernel developed by Erdogan et al. [42]. The stress intensity factors will reduce to:

$$
\begin{aligned}
& K_{\mathrm{III} L}=\frac{\sqrt{2}}{4} \mu\left(y_{L i}\right) \lim _{r_{L i} \rightarrow 0} \frac{w_{1 i}(\eta)-w_{2 i}(\eta)}{\sqrt{r_{L i}}}, \\
& K_{\mathrm{III} R}=\frac{\sqrt{2}}{4} \mu\left(y_{R i}\right) \lim _{r_{R i} \rightarrow 0} \frac{w_{1 i}(\eta)-w_{2 i}(\eta)}{\sqrt{r_{R i}}},
\end{aligned}
$$

where subscripts $L$ and $R$ designate the left and right crack tips, respectively, and the geometry of crack implies the following:

$$
\begin{aligned}
& r_{L i}=\left[\left(x_{i}(\eta)-x_{i}(-1)\right)^{2}+\left(y_{i}(\eta)-y_{i}(-1)\right)^{2}\right]^{\frac{1}{2}}, \\
& r_{R i}=\left[\left(x_{i}(\eta)-x_{i}(1)\right)^{2}+\left(y_{i}(\eta)-y_{i}(1)\right)^{2}\right]^{\frac{1}{2}} .
\end{aligned}
$$

By substituting Eq. (24) intoEq. (22) and the resultant equation into Eq. (25) after utilizing the Taylor series expansion of functions $x_{i}(\eta)$ and $y_{i}(\eta)$ in the vicinity of points $\eta= \pm 1$, the stress intensity factors can be expressed as follows:

$$
\begin{aligned}
& K_{\mathrm{III} L}=\frac{\mu\left(y_{L i}\right)}{2}\left[\left[x^{\prime}{ }_{i}(-1)\right]^{2}+\left[y^{\prime}{ }_{i}(-1)\right]^{2}\right]^{\frac{1}{4}} g_{z i}(-1), \\
& K_{\mathrm{III} R}=-\frac{\mu\left(y_{R i}\right)}{2}\left[\left[x^{\prime}{ }_{i}(1)\right]^{2}+\left[y^{\prime}{ }_{i}(1)\right]^{2}\right]^{\frac{1}{4}} g_{z i}(1) .
\end{aligned}
$$

\section{Results and discussion}

The main results of this study demonstrate that the stress intensity factors have been calculated for dif- 
ferent non-homogeneity parameters, crack length, and interactions between several cracks which are located at the interface between the nonhomogeneous layer and the isotropic half-plane. The above presented method allows for the consideration of dissimilar materials with multiple straight cracks subjected to anti-plane tractions. The results have been obtained through the instrumentality of distributed dislocation technique. The validity of the analysis is verified by considering a few examples, wherein the cracks are under constant normal traction.

The first problem is an interface crack in a functionally graded coating-substrate structure under the uniform anti-plane loading. External loading, $\sigma_{z y}=\tau_{0}$, is applied on the crack surface. The plots of dimensionless stress intensity factors for two different non-homogeneity parameters are drawn in Figure 2. In this case, excellent agreement is observed between the results of this study and those presented by Ding and $\mathrm{Li}[9]$. The second example deals with periodic interface cracks with three different distances between cracks centers, as shown in Figure 3. The cracks' faces are subjected to uniform anti-plane shear loads. The results favorably match results of Ding and Li [9].

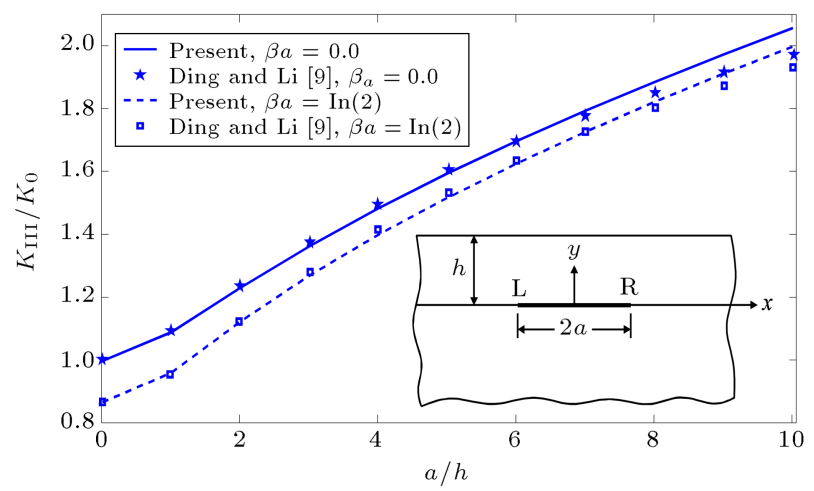

Figure 2. Comparison of the normalized stress intensity factors for straight crack in the interface under a uniform load.

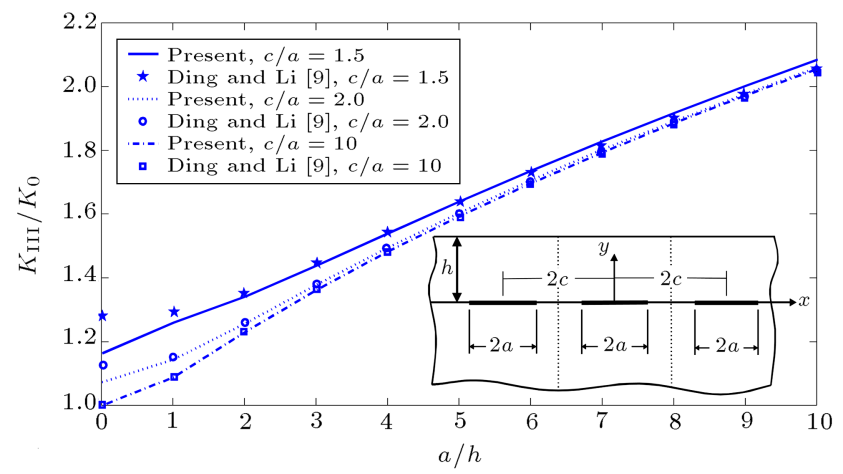

Figure 3. Comparison of the normalized stress intensity factors for periodic cracks in the interface under a uniform load.
The applicability of the methodology developed here is illustrated by solving some new problems.

In all remaining examples, since the medium is under concentrated anti-plane point loads as specified in Eq. (16), the quantity for making stress intensity factors dimensionless is $K_{0}=\tau_{0} / \sqrt{a}$ where $a$ is the half length of an embedded crack. In addition, it should be mentioned that the dimensions of distance between multiple cracks are taken $c / a=1.125$ as in all numerical results presented in this paper unless otherwise stated.

The effects of crack length and non-homogeneity parameter on the normalized stress intensity factors are given in Figure 4. The symmetry of the problem with respect to $y$-axis implies that the stress intensity factors at crack tips are identical. As physically expected, the stress intensity factors increase with the increase of crack length. Moreover, it can be observed that the normalized stress intensity factors increase as the non-homogeneity parameter rises. The effect of nonhomogeneity parameter on stress intensity factors is depicted in Figure 5. It can be seen that the highest value of dimensionless stress intensity factors occurs where the non-homogeneity parameter increases. In general, the influence of the coating thickness on the

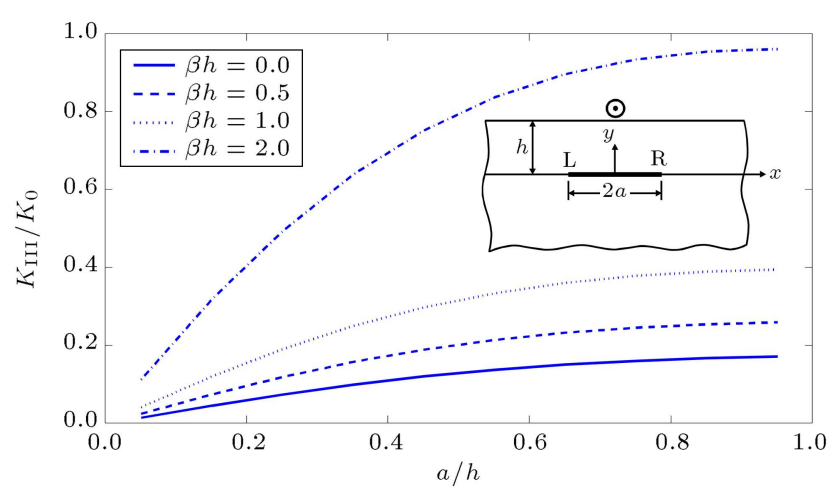

Figure 4. Normalized stress intensity factors for an interface crack versus the dimensionless crack length for different non-homogeneity parameters.

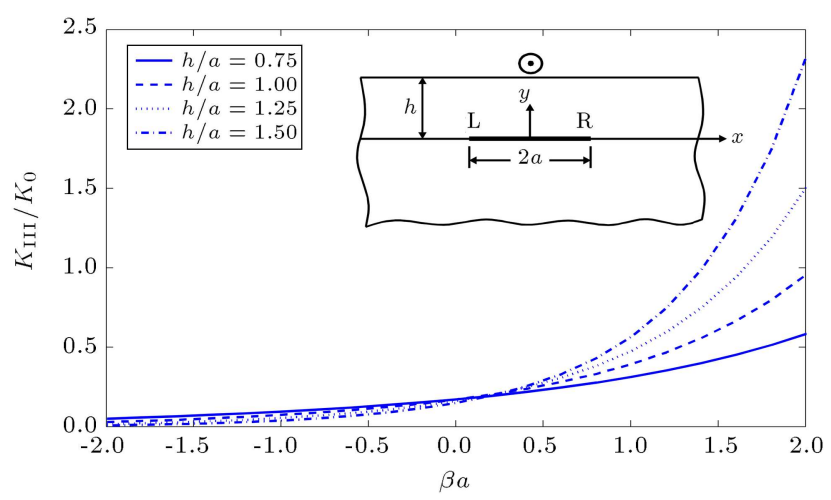

Figure 5. Normalized stress intensity factors for an interface crack versus the non-homogeneity parameter. 


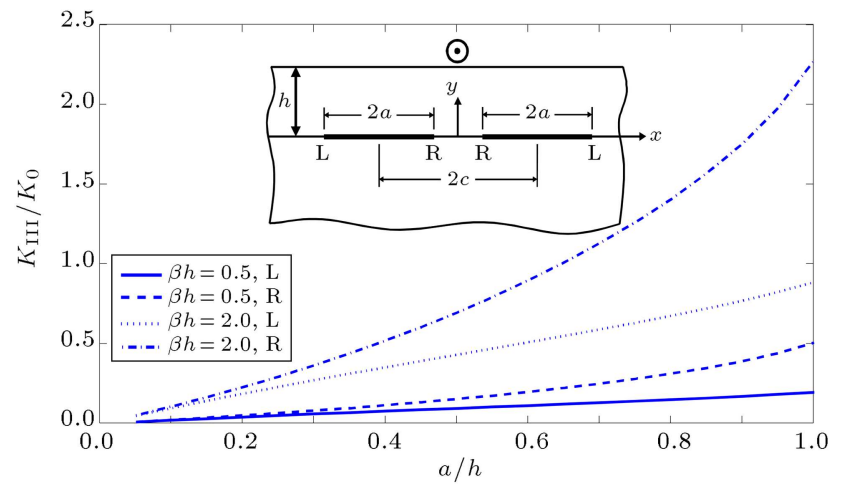

Figure 6. Normalized stress intensity factors for two interface cracks versus the dimensionless crack length.

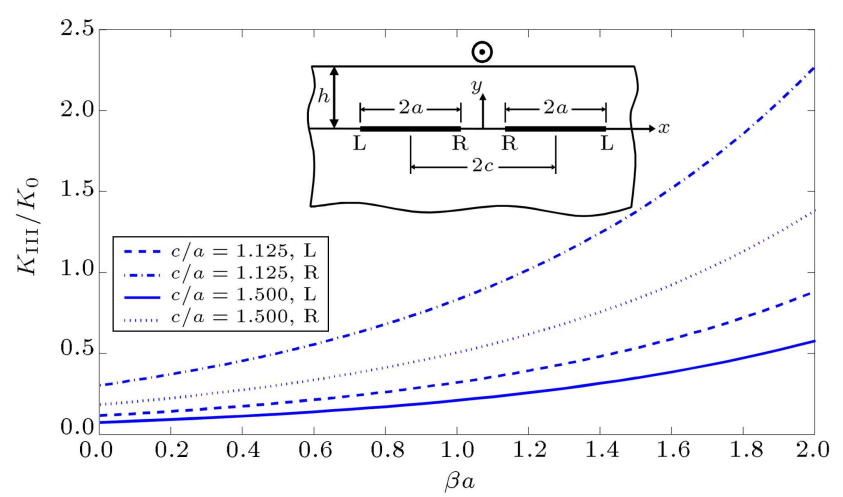

Figure 7. Normalized stress intensity factors for two interface cracks versus the non-homogeneity parameter.

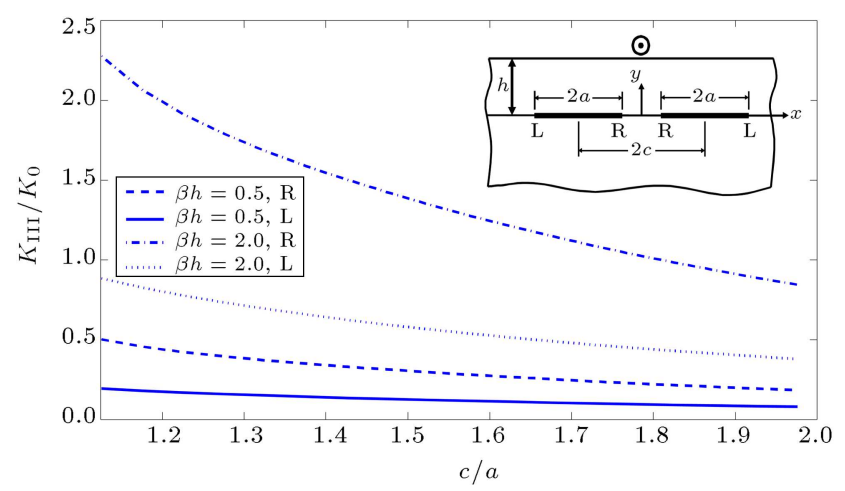

Figure 8. Normalized stress intensity factors for two interface cracks versus the dimensionless distance between the cracks.

stress intensity factors is as significant as that of the non-homogeneity parameter. Figure 6 shows the effects of non-homogeneity parameter and cracks' lengths between two interface cracks on the normalized stress intensity factors. It can be seen that an increase in either $\beta a$ or $a / h$ causes an increase in the stress intensity factors. As observed, dimensionless stress intensity factors for the two approaching crack tips change rapidly.

Two equal-length interface cracks are considered, as shown in Figures 7 and 8 . The lengths of cracks

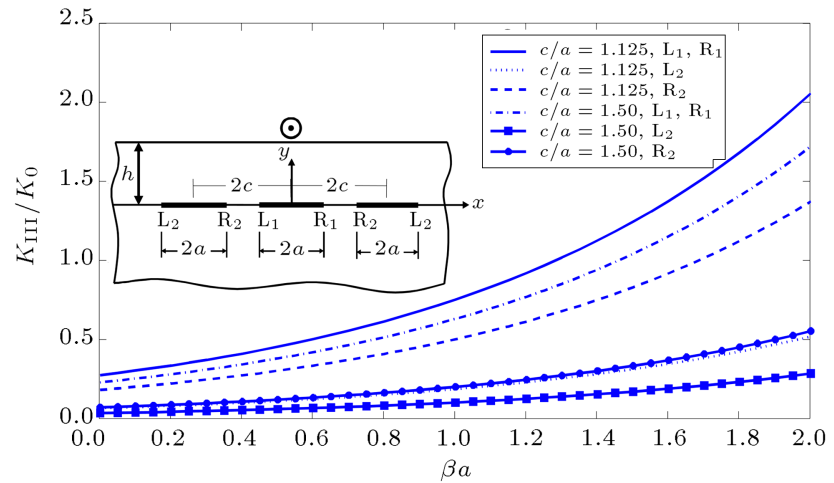

Figure 9. Normalized stress intensity factors for three interface cracks versus the non-homogeneity parameter.

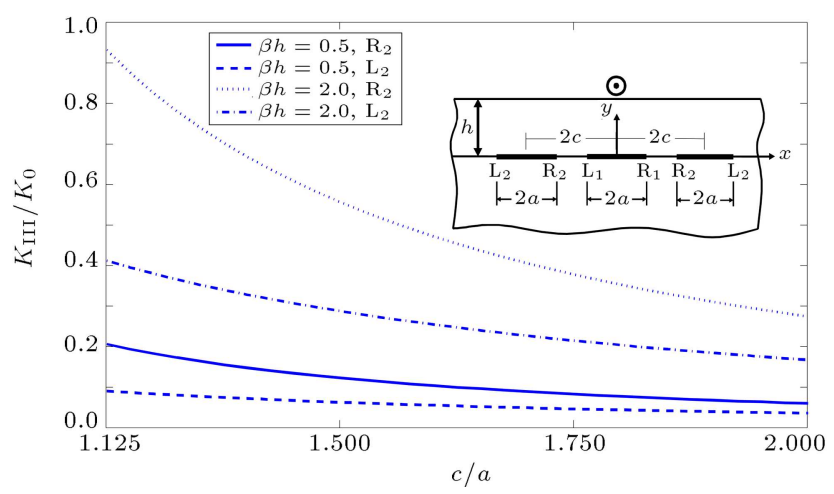

Figure 10. Normalized stress intensity factors for three interface cracks versus the dimensionless distance between the cracks.

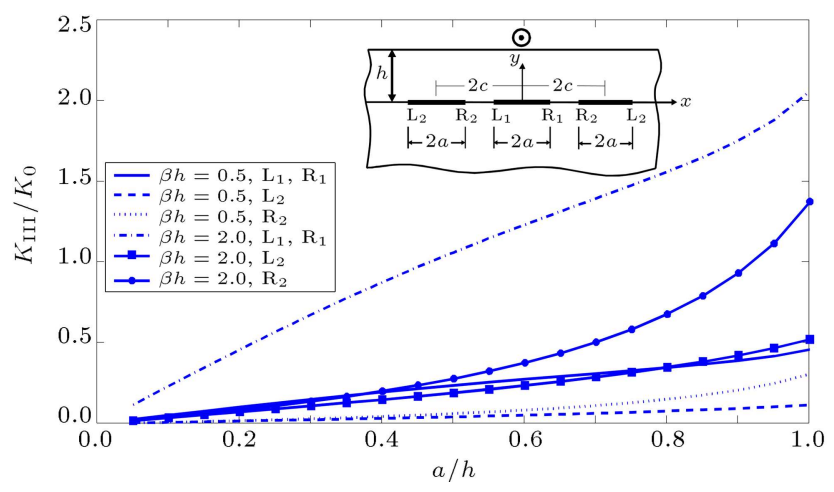

Figure 11. Normalized stress intensity factors for three interface cracks versus the dimensionless crack lengths.

remain fixed, while the non-homogeneity parameter and crack centers are changing. From Figures 7 and 8 , we may conclude that the stress intensity factors of the two interface cracks are mainly affected by the non-homogeneity parameter and interactions of cracks. According to the given results in Figure 8, it is evident that the normalized stress intensity factors decline steadily, while the distances between cracks increase as expected, due to the dramatic decline of the interaction between cracks. The final set of results, shown in Figures 9-11, presents stress intensity 


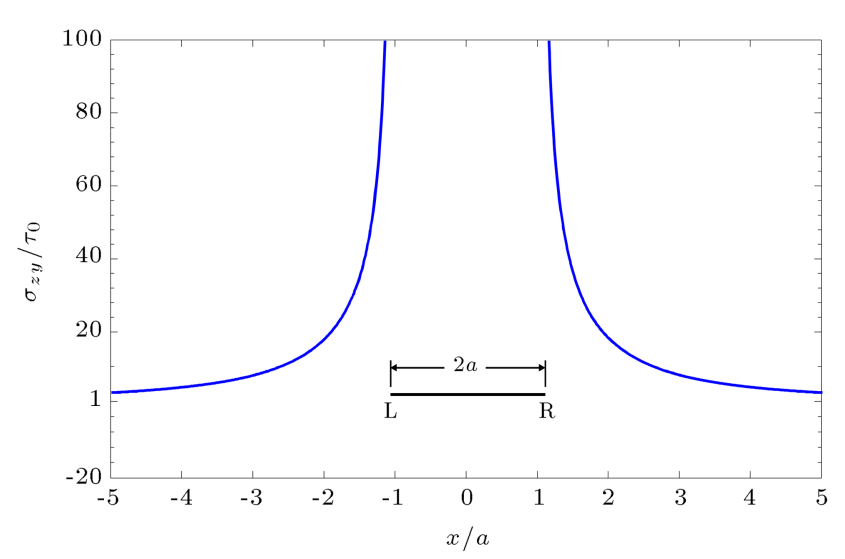

Figure 12. Normalized stress distribution around the crack tips.

factors for three interface cracks with changing nonhomogeneity parameter. Figure 11 shows the stress intensity factors of three interface cracks as a function of the crack lengths. Note that the stress intensity factors are highly influenced by the non-homogeneity parameter in which the stress intensity factors go up as non-homogeneity parameter rises.

Last but not least, stress distribution along $x$-axis is depicted in Figure 12 while $\beta a=2.0$. Singularities are, obviously, in the vicinity of the crack tips. It is crystal clear that the stress values plummet dramatically to be equal to the far field loadings at the sufficient distance of the crack tips.

\section{Conclusions}

The fracture behavior of a non-homogeneous layer along with a half plane bonded together was investigated. It was assumed that the half plane is homogeneous and the layer is non-homogeneous, in which the elastic properties are continuous throughout the layer and have discontinuous derivatives along the interface. The dislocation solution was obtained in a functionally graded layer and in half-plane containing Volterra-type screw dislocation by means of Fourier transformation. The dislocation solution was utilized to derive integral equations for the layer weakened by multiple cracks. The conclusions can be made as follows:

1. The stress intensity factors are highly influenced by the non-homogeneity parameter;

2. It was observed that as the crack length increases, the stress intensity factors escalate;

3. For the multiple interface cracks, the stress intensity factors are highly affected by the cracks' length and distance;

4. Negative values for the non-homogeneity parameters tend to decrease the stress intensity factor.

5. It was seen that decreasing the value of coating thickness while keeping $\beta$ constant tends to increase the stress intensity factor for negative values of parameter $\beta$.

\section{References}

1. Niino, M.H.T. and Watanabe, R. "The functionally gradient materials", Journal of Japan Society for Composite Materials, 13, pp. 257-264 (1987).

2. Erdogan, F. "Fracture mechanics of functionally graded materials", Composites Engineering, 5(7), pp. 753-770 (1995).

3. Jin, Z.H. and Batra, R.C. "Some basic fracture mechanics concepts in functionally graded materials", Journal of the Mechanics and Physics of Solids, 44(8), pp. 1221-1235 (1996).

4. Ming-Che, L. and Erdogan, F. "Stress intensity factors in two bonded elastic layers containing cracks perpendicular to and on the interface-I. Analysis", Engineering Fracture Mechanics, 18(3), pp. 491-506 (1983).

5. Delale, F. and Erdogan, F. "Interface crack in a nonhomogeneous elastic medium", International Journal of Engineering Science, 26(6), pp. 559-568 (1988).

6. Erdogan, F. and $\mathrm{Wu}, \mathrm{B}$. "Interface crack problems in layered orthotropic materials", Journal of the Mechanics and Physics of Solids, 41(5), pp. 889-917 (1993).

7. Erdogan, F., Kaya, A.C., and Joseph, P.F. "The mode III crack problem in bonded materials with a nonhomogeneous interfacial zone", Journal of Applied Mechanics, 58(2), pp. 419-427 (1991).

8. Lü, N.-C., Yang, D.-N., Cheng, Y.-H., and Cheng, J. "Asymmetrical dynamic propagation problems on mode III interface crack", Applied Mathematics and Mechanics, 28(4), pp. 501-510 (2007).

9. Ding, S.-H. and Li, X. "Anti-plane problem of periodic interface cracks in a functionally graded coatingsubstrate structure", International Journal of Fracture, 153(1), pp. 53-62 (2008).

10. Chen, Y.-J. and Chue, C.-H. "Mode III crack problems of two bonded functionally graded strips with internal cracks", International Journal of Solids and Structures, 46(2), pp. 331-343 (2009).

11. Chue, C.-H. and Yeh, C.-N. "Mode III fracture problem of two arbitrarily oriented cracks located within two bonded functionally graded material strips", Meccanica, 46(2), pp. 447-469 (2010).

12. Wan, Y., Yue, Y., and Zhong, Z. "A mode III crack crossing the magnetoelectroelastic bimaterial interface under concentrated magnetoelectromechanical loads", International Journal of Solids and Structures, 49(21), pp. 3008-3021 (2012).

13. Chao, C.K. and Lu, L.M. "Mode-III stress intensity factors of an arbitrarily oriented crack crossing interface in a layered structure", Journal of Mechanics, 29(04), pp. 643-651 (2013). 
14. Pan, S.-D., Zhou, Z.-G. and Wu, L.-Z. "Basic solutions of multiple parallel symmetric mode-III cracks in functionally graded piezoelectric/piezomagnetic material plane", Applied Mathematics and Mechanics, 34(10), pp. 1201-1224 (2013).

15. Ding, S.-H. and Li, X. "The collinear crack problem for an orthotropic functionally graded coating-substrate structure", Archive of Applied Mechanics, 84(3), pp. 291-307 (2014).

16. Choi, H.J. "Interfacial fracture analysis of bonded dissimilar strips with a functionally graded interlayer under antiplane deformation", Mechanics Research Communications, 78, Part (B) pp. 93-99 (2016).

17. Ordyan, M.G. and Petrova, V.E.E. "Interaction of cracks in an elastic two-component material under anti-plane shear loading", Science Journal of Volgograd State University. Mathematics. Physics, 3, pp. 53-62 (2016).

18. Rogowski, B. "Exact solution for an anti-plane interface crack in piezoelectro-magneto-elastic bimaterials", Archive of Applied Mechanics, 87(4), pp. 593-606 (2017).

19. Lapusta, Y., Onopriienko, O., and Loboda, V. "An interface crack with partially electrically conductive crack faces under antiplane mechanical and in-plane electric loadings", Mechanics Research Communications, 81 pp. 38-43 (2017).

20. Lü, N.-C., Li, X.-G., Cheng, Y.-H., and Cheng, J. "Asymmetrical dynamic propagation problem on the edges of mode III interface crack subjected to superimpose loads", Journal of Mechanics, 29(02), pp. 319-326 (2013).

21. Hu, K., Chen, Z., and Fu, J. "Moving dugdale crack along the interface of two dissimilar magnetoelectroelastic materials", Acta Mechanica, 226(6), pp. 20652076 (2015).

22. Monfared, M.M. and Ayatollahi, M. "Cracking in orthotropic half-plane with a functionally graded coating under anti-plane loading", Acta Mechanica Solida Sinica, 28(2), pp. 210-220 (2015).

23. Shin, J.W. and Kim, T.-U. "Transient response of a mode III interface crack between piezoelectric layer and functionally graded orthotropic layer", International Journal of Solids and Structures, 90, pp. 122128 (2016).

24. Yang, W.-Y., Zhang, S.-Q., Li, J.-L. and Ma, Y.-L. "Interface crack problems for mode II of double dissimilar orthotropic composite materials", Applied Mathematics and Mechanics, 30(5), pp. 585-594 (2009).

25. Cheng, Z., Gao, D., and Zhong, Z. "Interface crack of two dissimilar bonded functionally graded strips with arbitrary distributed properties under plane deformations", International Journal of Mechanical Sciences, 54(1), pp. 287-293 (2012).

26. Bhattacharya, S., Singh, I.V., Mishra, B.K., and Bui, T.Q. "Fatigue crack growth simulations of interfacial cracks in bi-layered FGMs using XFEM", Computational Mechanics, 52(4), pp. 799-814 (2013).
27. Ding, S.-H., Zhou, Y.-T., and Li, X. "Interface crack problem in layered orthotropic materials under thermo-mechanical loading", International Journal of Solids and Structures, 51(25), pp. 4221-4229 (2014).

28. Fan, M., Yi, D.K., and Xiao, Z.M. "Elastic-plastic stress investigation for an arc-shaped interface crack in composite material", International Journal of Mechanical Sciences, 83 pp. 104-111 (2014).

29. Zhao, W.-B., Zhang, X.-X., Cui, X.-C., and Yang, W.$\mathrm{Y}$. "Analysis of stress intensity factor in orthotropic bimaterial mixed interface crack", Applied Mathematics and Mechanics, 35(10), pp. 1271-1292 (2014).

30. Bui, T.Q. and Zhang, C. "Extended finite element simulation of stationary dynamic cracks in piezoelectric solids under impact loading", Computational Materials Science, 62 pp. 243-257 (2012).

31. Sharma, K., Bui, T., Zhang, C., and Bhargava, R. "Analysis of a subinterface crack in piezoelectric bimaterials with the extended finite element method", Engineering Fracture Mechanics, 104, pp. 114-139 (2013).

32. Liu, P., Yu, T., Bui, T.Q., and Zhang, C. "Transient dynamic crack analysis in non-homogeneous functionally graded piezoelectric materials by the X-FEM", Computational Materials Science, 69, pp. 542-558 (2013).

33. Liu, P., Yu, T., Bui, T.Q., Zhang, C., Xu, Y., and Lim, C.W. "Transient thermal shock fracture analysis of functionally graded piezoelectric materials by the extended finite element method", International Journal of Solids and structures, 51(11), pp. 2167-2182 (2014).

34. Yu, T., Bui, T.Q., Liu, P., Zhang, C., and Hirose, S. "Interfacial dynamic impermeable cracks analysis in dissimilar piezoelectric materials under coupled electromechanical loading with the extended finite element method", International Journal of Solids and Structures, 67-68, pp. 205-218 (2015).

35. Bui, T.Q. "Extended isogeometric dynamic and static fracture analysis for cracks in piezoelectric materials using NURBS", Computer Methods in Applied Mechanics and Engineering, 295, pp. 470-509 (2015).

36. Bui, T.Q., Hirose, S., Zhang, C., Rabczuk, T., Wu, C.-T., Saitoh, T., and Lei, J. "Extended isogeometric analysis for dynamic fracture in multiphase piezoelectric/piezomagnetic composites", Mechanics of Materials, 97, pp. 135-163 (2016).

37. Doan, D.H., Bui, T.Q., Duc, N.D., and Fushinobu, K. "Hybrid phase field simulation of dynamic crack propagation in functionally graded glass-filled epoxy", Composites, Part B: Engineering, 99, pp. 266-276 (2016).

38. Wang, Y. and Waisman, H. "Material-dependent crack-tip enrichment functions in XFEM for modeling interfacial cracks in bimaterials", International Journal for Numerical Methods in Engineering, pp. 1-24 (2017). 
39. Itou, S. "Stress intensity factors for four interfaceclose cracks between a nonhomogeneous bonding layer and one of two dissimilar elastic half-planes", European Journal of Mechanics-A/Solids, 59, pp. 242-251 (2016).

40. Monfared, M.M., Ayatollahi, M., and Bagheri, R. "Inplane stress analysis of dissimilar materials with multiple interface cracks", Applied Mathematical Modelling, 40, (19-20) pp. 8464-8474 (2016).

41. Hills, D.A., Kelly, P.A., Dai, D.N. and Korsunsky, A.M., Solution of Crack Problems: The Distributed Dislocation Technique, Kluwer Academic Publishers, (1996).

42. Erdogan, F., Gupta, G.D. and Cook, T.S. "Numerical solution of singular integral equations", In: G.C. Sih (Ed.) Methods of Analysis and Solutions of Crack Problems: Recent Developments in Fracture Mechanics Theory and Methods of Solving Crack Problems, Springer Netherlands, Dordrecht, pp. 368-425 (1973)

\section{Biographies}

Reza Sourki is a graduate student in Mechanical Engineering from University of Zanjan, Iran. He has received his $\mathrm{MSc}$ and $\mathrm{BSc}$ degree (both with honors) from University of Zanjan and has been awarded as the 1st ranked student. He, also, is a member of Exceptional Talents at the university as well as National Elites Foundation of Iran. His current areas of interest include theoretical fracture mechanics and vibrations.

Mojtaba Ayatollahi was born in Zanjan, Iran in 1977. He received his $\mathrm{PhD}$ degree from the Amirkabir University of Technology in 2007. He joined the Department of Mechanical Engineering, University of Zanjan, as an Assistant Professor in 2007; he became an Associate Professor in 2011 and a Professor in 2016. His current research interests include applied mathematics, elasticity, and theoretical fracture mechanics. He published over 40 papers in some wellknown journals since 2009 .

Mojtaba Mahmoudi Monfared received his $\mathrm{PhD}$ degree from the University of Zanjan. He is an Assistant Professor of Practice in Mechanical Engineering. His research interests include mixed boundary value problems, singular integral equations, solid mechanics, and fracture mechanics. 